\title{
La Interinstitucionalidad en las Relaciones Multilaterales de la Cooperación Interregional en América Latina
}

\author{
Andrés Rosales Segura*
}

\section{Resumen}

La interinstitucionalidad es una forma distinta de entender la cooperación, representa la actividad conjunta entre instituciones, tanto estatales como internacionales, tomando como base los objetivos de integración y cooperación regional latinoamericanos, es decir situar la interinstitucionalidad desde el punto en donde se difumina la política exterior y la política interna.

Este tema se ubica precisamente en esta disyuntiva de fronteras internas y externas, dejando de lado los temores de las injerencias o discusiones soberanas, y centrándose más en temas conjuntos, a través de estructuras democráticas y permanentes en el tiempo. Ello parece ser más práctico que las tradicionales decisiones unilaterales o de la situación particular del momento, incentivada por razones políticas o económicas en la mayoría de los casos.

Palabras claves: interinstitucionalidad, cooperación, regionalismo, innovación institucional, perspectivas.

\begin{abstract}
Interinstitutionality is a different way of understanding cooperation, represents the joint activity between institutions, both state and international, based on the objectives of Latin American regional integration and cooperation, which means, locate interinstitutionality from the point where foreign policy and internal politics are blurred.

This subject is precisely the disjunctive between internal and external borders, leaving aside the fears of interference or sovereign discussions, and focusing more on joint issues, through democratic and permanent structures over time. This seems to be more practical than traditional unilateral decisions or the situation of the moment, encouraged for political or economic reasons in most cases.
\end{abstract}

Keywords: interinstitutionality, cooperation, regionalism, institutional innovation, perspectives.

\footnotetext{
* MA in International Relations, Pontifica Universidad Católica de Valparaíso (PUCV), Chile. This article is based on the master's thesis. Lead researcher at International Consultants-Olympo SpA, Valparaíso, Chile. Email: andresrosales87@hotmail.com/ olympoconsultores@gmail.com. Received: October 19th, 2018; accepted: January 3rd, 2019.
} 


\section{Introducción}

Existen diferencias en la comprensión del término institución, esto derivado de las disciplinas, los autores, las coyunturas y los matices de su uso o descripción. Para este trabajo la institución parte de un sentido estatal, de la administración pública, contrastado en el marco de las relaciones internacionales. La institución es en muchos casos el eje del Estado, es el pilar que forma parte integral de la estructura gubernamental, aunado a esto, la institución representa la legalidad de un ente o autoridad reconocido en el Estado, esto fácilmente se replica en otros sistemas políticos en el mundo, que en conjunto legitiman sus propias aspiraciones a través de "instituciones internacionales" creadas para depositar en ellas compromisos internacionales, en ese sentido la dicotomía entre instituciones nacionales e internacionales, genera cierta confusión. Este análisis evidencia la dinámica entre instituciones de distinto orden y bajo diferentes disciplinas llevadas al campo de la cooperación en el continente americano.

No obstante, el entendimiento de dicho análisis requiere estudiar parte por parte y encontrar el punto en el que convergen instituciones nacionales e internacionales. Aislar el concepto “institución”, sin distinción de origen o propósito, encontrar ese punto de convergencia requiere de un esquema complejo que reúne a las instituciones en una interacción fluida y continuada, lo que se conoce como interinstitucionalidad. Esto es la forma en la que los Estados utilizan las herramientas duraderas en el tiempo y permiten depositar en ciertos órganos, el objetivo por los cuales son creadas y dotadas de recursos.

Desde la administración pública, la institución estatal surge con los procesos que comienzan desde el ejecutivo hasta la población. Dentro del Estado moderno y siguiendo la tradición de la política pública, es el medio por el cual el Estado logra encaminar acciones que permiten el resguardo, la seguridad y el bienestar de la población a través de un aparato gubernamental complejo y elaborado, que con el tiempo ha evolucionado y se ha adecuado a la coyuntura que vive cada país.

Por tanto, la discusión central es entender dónde comienzan unas instituciones y dónde terminan otras, en el sentido de conocer el flujo de acciones encaminadas a objetivos definidos, aún sin distinguir específicamente de dónde emanan dichos objetivos. El resultado varía en función de la continuidad de las políticas que fluyan de un sentido a otro (de lo nacional a lo internacional, y viceversa), es decir, como afectan de manera positiva los logros alcanzados en las instituciones internacionales a favor de la población de los países miembros, y si existe en la actualidad cooperación real entre instituciones, que, si bien son disímiles, pueden ser complementarias.

En este sentido las instituciones forman parte del Estado, tomando en consideración la política interna y exterior, ambas políticas funcionan para responder a los objetivos que la población. En las instituciones de orden interno, la administración pública al tener la capacidad para proponer, implementar y evaluar políticas públicas está comprometida con las políticas sociales y de sostenibilidad que impulse el Estado. Dichas instituciones nacionales se ven impactadas por el funcionamiento de la administración y la política estatal. En el ámbito internacional, las instituciones son moldeadas por la política exterior, al mismo tiempo que la política exterior es también parte de la política interna desarrollada en un espacio fuera del Estado, de ahí su vinculación entre disciplinas que determinan el tipo de institución y la función que tiene en su ámbito de acción. El vínculo que une a todo este esquema es el ejecutivo, el presidente a través de sus facultades y su carácter de actor tradicional de las relaciones internacionales. 
El vínculo presidencial, mediante sus atribuciones, permite establecer relaciones con los demás países ${ }^{1}$. En América Latina, al ser sistemas presidencialistas en su mayoría, las responsabilidades de la política exterior recaen en la Cancillería, Ministerio, o Secretaría de Relaciones Exteriores, dependiendo del nombre que se le dé en cada país, siendo esta la figura que coordina lo que el Poder Ejecutivo planea como políticas públicas en el ámbito internacional. El ejecutivo por su parte tiene la responsabilidad dual de administrar el orden interno y establecer acciones en lo internacional, con el objetivo de velar por la seguridad y los intereses nacionales, su actuación define en gran medida la conformación de relaciones y ejercicios con otras naciones que se encuentran en situaciones similares o con las cuales existe un especial interés por interactuar.

La relación entre el ámbito interno y externo que emana desde los objetivos del ejecutivo los realiza la interinstitucionalidad. Esto es entendido como una nueva generación de redes que pretende funcionar desde objetivos claros y determinados, en la cual, todas las dependencias de gobierno requieren tener contactos con sus pares dentro del Estado. Esto es particularmente relevante cuando hay temas que superan su quehacer diario, entendiendo que no se puede prescindir de una mirada multisectorial e interinstitucional. Por ejemplo, en el ámbito educacional, cuando se habla de intercambios estudiantiles para el mejoramiento del marco académico y la óptima preparación de estudiantes con una visión global.

Las experiencias nacionales propician una coordinación óptima y requieren de aquellas estructuras ya exploradas, para encontrar formas menos examinadas y otorgar la oportunidad de incorporarse a un esquema de aprovechamiento más amplio, cuyas instituciones ya están establecidas dentro y fuera del Estado. También permite adentrarse en otras situaciones que beneficien a las poblaciones a las cuales se dirigen dichas acciones, hacia un desarrollo integral de todo el andamiaje institucional del que cuenta el Estado y de los cuales los países ponen una apuesta para establecer contactos duraderos entre ellos.

Este trabajo está motivado por la creciente tendencia de conocer los procesos de integración dentro de Latinoamérica. En un momento que permite una mayor actuación de los países, su forma de establecer vínculos y esquemas complementarios, cuyos objetivos finales, también encuentran concordancia en mejorar las condiciones propias y a nivel regional. Dado lo anterior, resulta la hipótesis que guía este análisis enfocado a los procesos de integración, evaluar la interinstitucionalidad como una forma más profunda de compromisos y acciones entre países que tienen objetivos comunes. Aun así existe una imprecisión en la coordinación entre instituciones de carácter nacional e instituciones de integración latinoamericana. Por tanto se requiere una relación y coordinación estrecha, duradera y bien planeada, que vaya de objetivos regionales a acciones interinstitucionales estatales, tomando en cuenta que todos los Estados tienen estructuras internas definidas, constantes en el tiempo y consolidadas, evitando que las coyunturas de inestabilidad y la incertidumbre política y económica regional, detengan el avance y progreso de los proyectos de desarrollo, dando al ejecutivo un papel de coordinador y motor de objetivos que partan de lo general y puedan aprovecharse de mejor forma en lo particular.

\section{El papel del presidencialismo en la relación institucional interna e internacional}

Cabe resaltar que el poder presidencial en América Latina tiene muchas atribuciones en comparación con otros sistemas existentes en el mundo, y es el vínculo e hilo conductor entre la política interna y la política

\footnotetext{
${ }^{1}$ Esto hace referencia específicamente a América Latina.
} 
internacional. $\mathrm{Al}$ mismo tiempo es dirigente y figura trascendental de las instituciones internacionales y las instituciones nacionales. Siendo el actor tradicional de las relaciones internacionales y la figura representativa del Estado, su participación en los procesos de integración y en el seguimiento del Estado de Derecho, es indiscutible. La voluntad e injerencia que tiene en estas instituciones son de vital importancia para su progreso o deterioro. La contribución que puede entregar el Estado central (poder ejecutivo) está fuertemente ligada a compromisos políticos, tendencias políticas y periodos de gestión particulares en cada país de América Latina. Así que la cuestión es ¿qué pasa cuando la figura del ejecutivo cambia constantemente en comparación con las instituciones que son duraderas en el tiempo?

La historia latinoamericana nos ha servido de ejemplo para ver las tendencias que responden a esta interrogante, en la mayoría de los casos, no existe mayor distinción entre un periodo u otro. Sin embargo, sí se puede apreciar un enfoque distinto, dependiendo de las coyunturas nacionales o internacionales y del interés que tengan de enfatizar procesos de integración. En el caso particular de América Latina, la figura del presidencialismo está muy arraigada a la cultura política de la región, el poder y el centralismo que representa juega un doble papel para muchos analistas en la valoración positiva y negativa de su función. Dentro del Estado, el presidencialismo es el centro de las políticas públicas. Las acciones en el campo interno e internacional están sujetas a pasar por una estructura burocrática compleja, lo que en ciertos casos dificulta una contribución real, sobre todo en lo que concierne en cooperación internacional, al anteponer los intereses nacionales y coyunturas políticas internas.

Para muchos analistas, el presidencialismo tiene demasiadas atribuciones de poder, por lo cual algunos lo consideran un sistema precario. Duverger (1970) evidencia en el contexto latinoamericano la figura del presidencialismo, el cual "constituye una aplicación deformada del régimen presidencial clásico, por debilitamiento de los poderes del Parlamento e hipertrofia de los poderes del presidente: de ahí su nombre" (p. 152). En comparación con otras regiones en el mundo, en América existe predominancia del sistema político de corte presidencialista, y su reproducción en el continente permite que las instituciones tengan concordancia en los países dentro de la región, ya que siguen paradigmas de acción y procesos burocráticos similares. Evidentemente la cooperación internacional tiene una viabilidad de profundizarse en estructuras que buscan los mismos intereses y que tienen las mismas estructuras, de ahí el beneficio de un sistema tan centralizado que tiene la posibilidad de continuidad bajo ciertos parámetros.

La crítica central a este punto y en el cual cae en contrapeso es la voluntad política del dirigente en turno, dado que el centralismo en la figura del presidencialismo es mayor, principalmente en la política exterior, en comparación con otras estructuras de poder. Si bien otras instancias tienen participación, su poder de decisión es limitado, como lo es el caso del congreso que aprueba o rechaza lo que el ejecutivo propone o dispone. El poder judicial, en lo que respecta a la política exterior, tiene aún menos injerencia, ya que solo se limita a verificar que lo realizado esté en concordancia con lo que estipulan las leyes internas.

Por tanto, la política exterior en América Latina tiene su base en las formas presidencialistas de cada país. Según Bernales (1999) esta acción centralista de poder es "nociva" y "conduce generalmente al despotismo presidencial y a esa peculiar forma de gobernar que consiste en decidir sobre lo divino y lo humano, relativizando las funciones de los otros órganos del Estado y pasando por encima de toda definición constitucional de separación de poderes" (p. 162). Tener una política exterior con estas características hace que las instituciones creadas desde esa perspectiva guarden internamente algunos vicios propios del sistema político del cual emanan. 
Desde la perspectiva de los politólogos, el ejecutivo es en sí una institución, y su relación con otras instituciones tiene intrínsecamente una continuidad y permanencia en el tiempo. En ese sentido ningún presidente puede actuar arbitrariamente y dejar de lado la institucionalidad y los procesos políticos que ello conlleva. En él recae la responsabilidad de avanzar en procesos que están vinculados con las relaciones exteriores, haciendo que este dé prioridad a algunos temas y deje de lado otros que considere superfluos o improductivos en su momento.

La coordinación entre las instituciones y el ejecutivo es una relación que se debe analizar muy de cerca en el proceso de integración. La voluntad de integrarse puede verse obstaculizada por los recientes populismos en América Latina y los complejos surgimientos de políticos de ultraderecha, haciendo que se pierda el sentido real de la integración y la cooperación. Estas pueden pasar a un segundo plano de interés, tras objetivos particulares o promesas de campañas, con el objetivo de mantener una figura política popular, sin actuar de fondo en compromisos más ambiciosos o con miras a largo plazo.

Pese a que algunos autores consideran los sistemas presidenciales decadentes y poco adaptados a las necesidades de las sociedades latinoamericanas, no son opuestos a esquemas democráticos de participación y en muchos aspectos se hacen presentes actores que cada vez tiene mayor relevancia en la escena política internacional. Los propios parlamentarios trabajan para impactar de mejor forma a través de contactos con sus pares, se involucran e involucran a otros participantes, como la sociedad civil, académicos y demás agentes de opinión. Si bien la figura presidencial juega un papel central, actualmente tiene pesos y contrapesos que lo llevan a seguir objetivos comunitarios y cooperación en la región.

\section{Instituciones y multisectorialidad}

El esquema actual de la globalización ha contribuido a que los contactos entre pares institucionales puedan fluir de mejor forma cuando las relaciones entre los países son estables. Existe por tanto la necesidad de involucrar organismos similares, pero también sectores que confluyan. Surge con la idea de estructuras equiparables, la interinstitucionalidad como un esquema de acción entre instituciones del mismo país o de distintos países. Se habla de una coordinación entre pares, sea del ámbito legislativo, ejecutivo o incluso judicial, la cual pretende aprovechar las ventajas logísticas o experiencias de otras instituciones estatales.

El concepto de interinstitucionalidad hace referencia a la coordinación de actores, a la interacción de instituciones a través de mecanismos de acción conjunta en torno a proyectos comunes, a la formulación, construcción y ejecución colectiva de programas, proyectos y acciones que involucran iniciativas, recursos, potencialidades e intereses compartidos. La interinstitucionalidad constituye una alternativa de gestión relacional e interconectada de agentes interrelacionados, frente a modelos auto-centrados e individuales (Duque, 2011:3). Consiste en la integración de diversos objetivos particulares, a veces contradictorios y otros complementarios, que le permiten al país entrar en el mundo de la "competencia". Así, la interinstitucionalidad, como proyecto inacabado y por tanto en permanente construcción, constituye una alternativa relacional frente a los modelos de organización, gestión y desarrollo².

\footnotetext{
${ }^{2}$ Duque explica en su prólogo que la interinstitucionalidad constituye una alternativa de gestión de programas de formación avanzada frente a programas auto-centrados e institucionales. Como forma de acción conjunta, cooperativa, complementaria y solidaria, se perfila como una estrategia de gestión innovadora que empieza a consolidarse en muchos países del subcontinente
} 
Cuando se habla de interinstitucionalidad de manera interna, se refiere a la estructura gubernamental que por ley requiere de la cooperación de otras instituciones para complementar información o decisiones y concretar políticas públicas eficaces, en línea con las necesidades de la población. Esta forma, aún es poco estudiada y entendida desde el interior del país, es un elemento vinculatorio necesario y existente desde la estructura del Estado mismo, la colaboración entre instituciones va en función de mejorar las capacidades que cada una de ellas tenga para ejercer la función por la cual ha sido creada.

En la mayoría de los países se habla en los discursos políticos de dos elementos claves que se repiten continuamente: la intersectorialidad y la interinstitucionalidad, haciendo énfasis en la necesidad de desarrollarlos. Son aspectos complementarios, constantemente invocados, pero guardan muchas impresiones de cómo se deben entender y cómo se deben aplicar³. Uno de ellos remite a lógicas de acción colectiva, distinguiéndose así entre el sector público, el sector mercantil, el sector privado no mercantil o las comunidades.

En ese marco, la intersectorialidad alude a las relaciones entre tales sectores y cubre el estudio de las diferentes modalidades de asociación público-privada. Otro significado de sector remite más bien a especialidades del conocimiento que se expresan en el aparato gubernamental a través de la organización funcional por sectores, tales como educación, salud, agricultura, etc. La intersectorialidad, en este caso, se refiere en primera instancia a la relación entre diversos sectores gubernamentales (Cunill-Grau, 2013: 2). Objetivo de este trabajo es la interinstitucionalidad, por lo que no se abordará en mayor detalle la intersectorialidad.

\section{Interinstitucionalidad}

La interinstitucionalidad, concepto sobre el que centramos el análisis, está relacionado con el concepto anterior al tener una coordinación entre dependencias gubernamentales de un mismo Estado. Los fines por los cuales se da esta relación son diversos, no obstante, existe una necesidad de coordinación de todo el aparato gubernamental para su mejor funcionamiento. Ninguna institución tiene los recursos fiscales o personales, el conocimiento o el mandato legislativo para planear o proveer la cantidad de servicios esenciales en un proceso efectivo de planeación y transición. "Los acuerdos interinstitucionales implementados por equipos interinstitucionales productivos, organizados y versátiles son los fundamentos de un programa efectivo y cohesivo de transición" (Crame, Gramlich \& Peterson, 2004: 115). Todos estos aspectos se repiten en los países latinoamericanos, ya que las instituciones son equiparables, así como los temas que atienden. Por tanto, se requiere de una mayor coordinación entre ellas para resolver temas complejos, y que puedan mejorar las condiciones de la población en un menor periodo de tiempo.

Existen asimismo retos de la interinstitucionalidad que deben ser superados, ya que parte de la coordinación depende de las propias instituciones y sus relaciones. Se entiende que funcionan de manera

\footnotetext{
latinoamericano. Trabajo que sale del Doctorado Interinstitucional en Educación, en colaboración con: Universidad del Valle (UV) - Universidad Distrital (UD) - Universidad Pedagógica Nacional (UPN). Bogotá, Colombia. 2011.

${ }^{3}$ En el caso de la intersectorialidad, se reconoce esta condición de imprecisión respecto al concepto de "lo sectorial", que tiene distintos significados y, en consecuencia, también su noción. Dado que alude a diversos sectores gubernamentales en cuyo caso cambia de perspectiva dependiendo de la materia tratada, a sabiendas que si los problemas que buscan resolver son multicausales, la integración sectorial puede mejorar su abordaje integral.
} 
vertical dentro del aparato gubernamental, es decir poseen una jerarquía uniforme y subordinada al ejecutivo. Esto fija su nivel de responsabilidad respecto a las tareas que tienen que cumplir hacia adentro de su institución, donde el poder de decisión es autónomo para cada sector y donde existe un poder de negociación equivalente hacia las contrapartes.

La coordinación implica la posibilidad de mejorar la provisión de servicios, maximizar los recursos y aumentar la efectividad en la colocación de los bienes sociales. Esto implica la necesidad de que "un grupo de personas o instituciones logren trabajar juntas para lograr un objetivo" (Winer \& Ray, 1994; Butterworth et al, 2001) o para alinear metas, prioridades y recursos. El resultado de esta colaboración entre instituciones deja compromisos que forman parte de esta estructura ideal, lo que permite vincular de mejor forma a los organismos internos para trabajar de manera conjunta y atender problemas que por sí solos no pueden. Estos mecanismos para afianzar las relaciones entre instituciones se materializan en "acuerdos interinstitucionales", como la elaboración de memorias de entendimiento, lineamientos de trabajo interinstitucional, así como de mecanismos de monitoreo y seguimientos de avances para los cuales fueron creadas (Instituto Nacional de Desarrollo Social, 2011: 112).

La coordinación no es una obligación (legal) para las instituciones, por lo que su vinculación emana de los retos coyunturales, ya que en muchas ocasiones se dificulta que exista una real coordinación. La colaboración debe tener como meta el cumplimiento de los objetivos durante el proceso, y que este se mantenga en el tiempo. La idea es tener los menores contratiempos posible (sobre todo cuando se trata de trabajar directamente con la población) y especialmente, que se cumplan los propósitos definidos de manera colectiva. El proceso de coordinación además debe permitir la disminución de los costos asociados con la atención individual de cada una de las instituciones, la obtención e integración de información que pueda compartirse para determinar criterios de intervención en la población y así maximizar el uso de los recursos.

Las instituciones promueven múltiples programas en sus distintas áreas de trabajo (CEPAL, 2018) y que sólo se limitan a su campo de acción, sin involucrar al resto de las dependencias que tienen injerencia y que pueden proyectar mejor esas oportunidades para la población que lo requiera. En Chile existe el Comité Interministerial, que es presidido por el Ministro de Justicia y Derechos Humanos y lo integran los Ministerios del Interior, Relaciones Exteriores, Defensa, Secretaría General de la Presidencia, Desarrollo Social, Educación y el Ministerio de la Mujer y la Equidad de Género, que representan ese tipo de cooperación interinstitucional, cuyos objetivos son aprovechar las estructuras existentes y mejorar las condiciones en las que atienden los problemas. Esta aproximación es ejemplo de instituciones de carácter interno que trabajan de manera colaborativa para fines más ambiciosos.

Dentro del trabajo presentado por la OCDE, "Panorama de las Administraciones Públicas América Latina y el Caribe" (2017), se habla de la importancia de la coordinación interministerial, que representa la misma figura que la interinstitucionalidad y esta es entendida como una de las funciones claves que el estudio define como Centro de Gobierno $(\mathrm{CdG})^{4}$. En casi la totalidad de los países que conforman América Latina y el Caribe y que la OCDE toma como base para presentar sus análisis de la coyuntura

\footnotetext{
${ }^{4}$ El centro de gobierno (CdG) se definió como la organización y las unidades responsables por determinadas funciones transversales críticas (gestión estratégica, coordinación, monitoreo y mejora del desempeño, gestión política, comunicaciones y rendición de cuentas) relacionadas con la gestión de las principales prioridades del gobierno. Entre las unidades características figuran el ministerio o la secretaría general de la Presidencia, la oficina del primer ministro y la oficina del gabinete, aunque a veces esas funciones pueden ser desempeñadas por unidades adscritas a otras dependencias del gobierno (finanzas, planificación, presupuesto, etc.).
} 
actual, el papel del denominado CdG es facilitar o apoyar a los ministerios encargados de esas iniciativas, aunque también puede asumir un papel de liderazgo en casi la mitad de esos países. Es imprescindible para que el gobierno tenga la seguridad de responder de manera integral a problemas multisectoriales, así como para disminuir la duplicidad de tareas o posibles contradicciones en las políticas gubernamentales. Es por tanto que se requiere de un eje que pueda, al interior del Estado, coordinar como un "intermediario imparcial" entre los ministerios de línea, que tienen sus culturas burocráticas y sus programas sectoriales propios, alineándolos con la dirección general del gobierno. Además, el surgimiento de problemas complejos y multidimensionales que no se pueden abordar exclusivamente mediante respuestas ministeriales "verticales", ha puesto de manifiesto la importancia de una dirección y coordinación centralizadas (OCDE, 2017).

Estos llamados CdG, según la OCDE (2017), son críticos para organizar y apoyar el proceso de toma de decisiones del jefe de gobierno, primer ministro o presidente, para garantizar el logro de las prioridades gubernamentales. En 2015, la mayoría de los presidentes y primeros ministros de América Latina y el Caribe (ALC) se apoyaban en diferentes canales en la toma de decisiones. Las reuniones de gabinete ( $93 \%$ de los países) y los contactos bilaterales directos con los ministros (80\%), son los principales vehículos para tratar cuestiones de política, dejando atrás a los grupos de asesores, con presencia en solo un $46 \%$ de los países. El papel asignado a las reuniones de gabinete en ALC resulta digno de mención, tratándose de una región donde la mayoría de los países funcionan con sistemas presidencialistas en los cuales, tradicionalmente, se consideran inusuales las discusiones colectivas de gabinete.

Por tal motivo, la coordinación entre instituciones o dependencias gubernamentales ha venido cobrando cada vez mayor importancia, y eso se refleja tanto en el interior del país como fuera, donde se remarcan, en foros multilaterales, las virtudes de mejorar la coordinación entre estos organismos de orden interno, con la finalidad de ejecutar políticas públicas pensadas en involucrar a más organismos del orden público. El análisis de la OCDE muestra que en los últimos años 60\% de los países de ALC han indicado que el número de iniciativas interministeriales ha aumentado, en comparación con el 51\% de los países de la OCDE. El 33,3\% de los demás países de la región han indicado que el número de iniciativas interministeriales se ha mantenido estable (44\% en la OCDE), mientras que 6,7\% de ellos han indicado que dicha cifra se ha reducido ( $4 \%$ entre los países miembros de la OCDE).

En general, la coordinación se canaliza mediante comités interministeriales. En 80\% de los países de ALC, el CdG tiene la responsabilidad de organizar grupos o comités multisectoriales para la coordinación de políticas. Dichos comités existen a nivel ministerial en $80 \%$ de los países de América Latina y el Caribe (en comparación con 85,2\% en la OCDE), a nivel viceministerial en 40\% de los países de la región (70,3\% en la OCDE) y a nivel de dirección en $46,7 \%$ de esos países (74,1\% en la OCDE). Por lo tanto, en contraste con la práctica en los países miembros de la OCDE, en ALC, los comités interministeriales tienden a estar integrados por funcionarios de nombramiento político que por servidores públicos de alto nivel. Esto puede llevar a una falta de continuidad o de memoria institucional en la coordinación interministerial, ya que hay mayores índices de rotación de personal entre los funcionarios de nombramiento político que entre los servidores públicos de carrera. En la región la influencia de los CdG para promover la colaboración entre los ministerios varía de un país a otro. En el 33,3\% de los casos se considera que el CdG tiene una alta influencia sobre los ministerios de línea para alentar la coordinación (en comparación con 30\% en los países de la OCDE), lo que significa que puede llegar a imponerles consecuencias o sanciones. En el 46,7\% de los demás países de la región se indica que la medida de dicha influencia es moderada (59\% entre los países de la OCDE). 
Con lo anterior, se puede ver la manera tan sutil en que las instituciones de orden interno se entremezclan con la actividad internacional, y todo recae en los temas que atienden y la complejidad de resolver los retos que ellos generan de manera individual o aislada del resto de los organismos internos e internacionales. La línea delgada entre lo interno e internacional se difumina aún más por efecto de la cooperación internacional, la cual es entendida en un carácter que no conoce barreras, y todos los organismos e instituciones de cualquier orden tienen la capacidad de cooperar en gobiernos democráticos. Esta capacidad se vuelve una exigencia para mejorar la forma de acción y los logros que pretenden alcanzar.

Esto se encuentra en función de la centralidad de la política exterior hacia el Ejecutivo y en un segundo término para el poder legislativo. De cualquier forma, aún no existen protocolos específicos para encaminar los esfuerzos en contactos que vayan hacia una coordinación interinstitucional bilateral o multilateral. Entre estas relaciones interinstitucionales o interministeriales existe una que ha tenido un éxito muy profundo, las experiencias legislativas que se vinculan con otras instituciones pares, para debatir y compartir experiencias de éxito y fracaso con el objetivo de tener un mayor entendimiento y cooperación entre los países que entablan estos contactos. Los encuentros interparlamentarios representan un gran logro y alcance para conocer las posturas externas de los países vecinos o con los cuales están interesados en profundizar sus contactos.

\section{Los recursos interparlamentarios y vías interinstitucionales}

Los contactos interparlamentarios son un poco más evidentes desde la lógica de las instituciones, pero con la misma mecánica y logros alcanzados. En la diplomacia parlamentaria (Burgos, 2017: 95) se refleja la necesidad de establecer un conjunto de regímenes y organizaciones internacionales que permitan, tanto a nivel mundial y regional, coordinar acciones y políticas, aprender de las mejores prácticas, y establecer reglas aplicables a distintos campos de acción interdependientes. En ese sentido, el poder legislativo ha encontrado formas de entablar contactos con sus homólogos de otros países, con la intención de externalizar experiencias y ver la utilidad de aplicar algunos esquemas a sus respectivas realidades internas, así como mejorar las relaciones y confrontar asuntos que el poder legislativo, hasta hace poco, no había tenido en su agenda. Esta discusión de ideas ciertamente genera un entendimiento entre países vecinos, que inicialmente generan, a través de la diplomacia y la negociación, vínculos de confianza que facilitan la acción del ejecutivo al momento de recurrir a sus homólogos, donde los contactos parlamentarios ya han establecido pautas de diálogo. Con el paso del tiempo, cualquier acuerdo que se establezca con estas características estará en mejores condiciones para ser aplicado, y, por ende, traerá de facto un aprovechamiento real y duradero para la población de cada país.

Así como la diplomacia parlamentaria, los contactos entre instituciones de orden estatal se desarrollan en paralelo, siguiendo la política exterior del país y al mismo tiempo aprovechando las estructuras existentes, para maximizar los recursos y evitar la internacionalización de problemas internos. Por ejemplo, el narcotráfico y crimen organizado son problemas considerados transnacionales, y es claro que el efecto de dichos fenómenos es más acentuado en unos países que en otros, pero ninguno está exento de lidiar con estas dificultades. Por lo cual, se requiere una cooperación por parte de organismos especializados que buscan combatir y erradicar los efectos nocivos de estos problemas. 
El 30 de marzo de 2016, los Fiscales Generales y Procuradores Generales de América Latina se reunieron en Lima, Perú, con ocasión de la Cumbre de Fiscales Generales de América Latina, "Estrategias en la Lucha contra el Crimen Organizado, Corrupción y Lavado de Activos" (La Razón, 2016). En dicho encuentro se emitió una declaración, de la cual participaron Perú, Ecuador, El Salvador, Paraguay, República Dominicana, Chile, Brasil, Costa Rica, México y Uruguay; en la cual se establecieron las pautas de cooperación en información y mecanismos de asistencia técnica y capacitación, reiterando dos puntos: la necesidad de establecer vínculos entre instituciones de distintos países en temas que no respetan fronteras y dos, el hecho que los contactos son cada vez más frecuentes, pero poco conocidos como parte de la estructura del sistema internacional.

Estas vías son parte de lo que puede representar la interinstitucionalidad en ambos sentidos (en lo interno e internacional), haciendo que las instituciones sean más capaces de ejecutar sus funciones, y aprovechando los contactos existentes que el propio ejecutivo establece en su agenda internacional. No sólo estos vínculos pueden ser específicos entre instituciones homólogas, la era de la globalización nos está llevando a un plano aún más ambicioso: los contactos entre instituciones del Estado y las instituciones de carácter internacional o regional según sea la necesidad coyuntural (Allard, 2009).

\section{Esfuerzos colectivos}

La amplitud de contactos se multiplica en la medida en que se integran más actores o los países establecen en su política exterior atender ciertos temas que involucran a demás Estados vecinos o regiones con los que se fijan objetivos. Los procesos de integración en América Latina buscan profundizar los esfuerzos colectivos para mejorar aquellos aspectos en los que los países encuentran concordancias.

Para el esquema de integración latinoamericano, las experiencias europeas de unificación, específicamente la Unión Europea, influyeron en gran medida en la conformación de la red de bloques comerciales y políticos que existen en la actualidad. El éxito de la Unión Europea por mucho tiempo fue la guía para que los países latinoamericanos siguieran con el tradicional proceso integracionista desde la Teoría de la Integración Económica ${ }^{5}$, que tiene una suerte de secuencia en el proceso de conformación de bloques y estructuras que se instauran para dar legitimidad a los procesos económicos y poner las pautas para entablar una relación más profunda entre las partes. Cuando se establecen acuerdos, se crean organismos de los cuales los países se hacen miembros por voluntad propia, sabiendo que la cooperación genera un esfuerzo colectivo y aminora los costos de intentar resolver los problemas de manera individual. Cuando esto sucede se forman dos esferas que por mucho tiempo se analizaban de manera separada una de la otra, lo interno y lo internacional.

El factor globalización, que si bien no es nuevo (Wallerstein, 2016), solo está dado por ciclos económicos que van acompañados por momentos históricos. En la actualidad son más evidentes los actores nuevos y otros que toman más relevancia en la acción de las políticas internas e internacionales. El período de

\footnotetext{
${ }^{5}$ La integración económica inicialmente considerada como una segunda rama del estudio de la economía internacional. No es un fenómeno moderno: varios países europeos se construyeron a partir de un proceso integrador que eliminó las barreras que separaban distintos mercados regionales. En el decenio de 1950, el término de integración económica se consagra totalmente, su uso se difundió y el concepto es definitivamente establecido. Tamanes (1990: 170), considera que la integración económica "es un proceso a través de cual dos o más mercados nacionales previamente separados y de dimensiones unitarias estimadas poco adecuadas se unen para formar un solo mercado (mercado común) de una dimensión idónea”.
} 
los años 80’s y 90’s en América Latina, se caracterizó por ser una época de firmar acuerdos y tratados, sin tener una visión objetiva de la utilidad o servicio para la economía interna, existían algunos acuerdos clave que eran imprescindibles, pero en su generalidad, la apertura comercial latinoamericana, se vio plagada de tratados sin mucho alcance.

En la actualidad, los organismos de integración se han especializado a ciertas áreas u objetivos bien definidos, para evitar duplicidad y un gasto innecesario a los Estados. Evidentemente la carga económica que genera un organismo internacional es un costo que tiene que pagar la población de los Estados miembro y que en muchas ocasiones pone en tela de juicio su funcionalidad y su permanencia, dado que los objetivos no son cumplidos por organismos que dejan de funcionar o se congelan en el tiempo. Los problemas que intentaba resolver desde un inicio persisten o en el peor de los casos, se transforman en problemas aún más difíciles de resolver, corriendo el riesgo de propagarse en la zona o en países que tiene condiciones muy parecidas, haciendo con esto que se promueva la creación de nuevos organismos que combatan dichos problemas, entrando en una contradicción y complejidad que caracteriza el sistema de integración latinoamericano.

Haciendo una distinción entre los diversos organismos de integración latinoamericana, estos pueden ser clasificados dependiendo de su alcance: asociación económica, zona de libre comercio, unión aduanera, etc. ${ }^{6}$. Esta clasificación en muchos casos permitía ver el nivel de compromiso económico que tenían los países en cuestión, no obstante, la gran diversidad de temas y contactos políticos entre países permitieron el cauce de otro tipo de integraciones de corte política: foros de cooperación, cumbres y mecanismos de diálogo político, cuyo progreso permitió la consolidación de dichos contactos al punto de institucionalizarlos. La mezcla entre lo económico y lo político amplió aún más la variedad de organismos de integración latinoamericana, muchas de estas instituciones permanecen en el tiempo, otras tantas se han trasformado y algunas de ellos han quedado en el olvido.

En la bibliografía actual el foco de atención está dirigido hacia dos organismos en particular: Alianza del Pacífico y MERCOSUR. Sin embargo, a pesar de tener afinidades y objetivos económicos de cooperación y concertación económica en pro de la región, no son iguales en sus estructuras. El matiz distintivo que tienen permite hacer una comparación, tomando en cuenta sus orígenes, objetivos y particularidades que guarden distancia entre una y otra.

Estas peculiaridades se centran en la distinción entre los distintos bloques existentes, algunos de los cuales son difíciles de encuadrar en una clasificación concreta y específica, como es el caso del MERCOSUR, una "unión aduanera imperfecta" que tiene un origen y funcionamiento puramente comercial, pero sus avances y retrocesos están dados por la política centrada en los países miembros. Caso distinto a la Alianza del Pacífico, que, si bien tiene esta movilidad de bienes y capitales, no es un mercado común, ni una unión aduanera, es un organismo que busca profundizar la integración de servicios, capitales e inversiones a través de la voluntad política.

En definitiva, todos los organismos de integración tienen puntos en los cuales son equiparables, sea por sus objetivos, la coyuntura en la que fueron creados, su carácter predominante de acción o por los países que la conforman. Cada uno de ellos es distinto y han demostrado que pueden mantener una relación muy compleja entre ellos, sus miembros y otros organismos fuera de la esfera latinoamericana. Todo

${ }^{6}$ El pensamiento de la integración latinoamericana fue fomentado por las ideas de finales del siglo XVIII. Las teoría de la integración económica generalmente enmarcan de manera taxonómica el nivel de compromiso entre países: Área de Preferencias Comerciales, Zona de Libre Comercio, Unión Aduanera, Mercado Común, Unión Económica o Monetaria, Integración Total. 
sistema de integración en algún punto responde a objetivos de carácter político, económico, social y cultural, dado la coyuntura que se centre en los países que la conforman, de ahí la estabilidad o inestabilidad con la que actúan, que impacta directamente en la fortaleza o debilidad que tenga la institución.

\section{Cosmovisión de la institucionalidad}

En cuanto a la relación que existe entre las instituciones regionales e instituciones nacionales, existen tópicos o temas que ambos tratan, temas que son de alcance general y que atañen a más de un país, como: cambio climático, pobreza, subdesarrollo, mejoramiento en infraestructura y progreso económico. En todos los casos fácilmente se puede pensar que instituciones dentro y fuera del país tocan estos puntos, pero su relación es muy particular. Si por ejemplo tocamos el tema de salud y bienestar, o el tema de hambre y pobreza, vemos que podemos dimensionar que todo tipo de instituciones preocupadas por erradicar o aminorara esta problemática llevan a cabo labores específicas con objetivos definidos. No obstante, la escala de acción es muy distinta para cada tipo de instituciones. Para temas como los antes mencionados, en el orden interno se activan una serie de políticas públicas que plantean este problema en lo local. Para las instituciones internacionales las acciones son a través de compromisos por parte de las autoridades estatales, que se aterrizan en lo nacional y generalmente se convierten en reglamentos, leyes o normas de adopción legislativa. Para llegar a este punto se debe de pasar conscientemente del ámbito económico al político, los compromisos que se generan en tratados internacionales no son nada sin la voluntad de los países por efectuarlos, esta voluntad está encaminada a ser política, aunque la motivación en la mayoría de los casos sea económica.

Las instituciones internacionales indudablemente dependen de la voluntad de los países para reaccionar, y la forma de ejecutar dichos compromisos es a través de la vasta infraestructura gubernamental de cada país. La peculiaridad más grande es la forma en la que coexiste ambos tipos de instituciones, los tópicos o problemas nacen en el interior de los países, pero encuentran concordancia en lo internacional. Cuando hay relación entre países, por contactos económicos, encuentran que hay problemas comunes; una vez identificados estos, se dedican a debatir y dialogar para generar compromisos conjuntos, esos compromisos recaen en los organismos internos. Respecto de la coordinación interinstitucional, cuando encuentran temas internos que son difíciles de solucionar, la demanda social pone en la mira de la opinión pública la forma en la que el Estado puede reaccionar, por ende, se genera desde el presidente o más concretamente en Cancillería, el establecimiento de las "Agendas", como mecanismos prioritarios que son discutidos en un alto nivel político y ponen en acción los mecanismos de coordinación y cooperación institucional.

Todo nos lleva a concluir que los organismos de integración regional, al ser estructuras creadas a partir de voluntad política de los países, a través de gestiones establecidas por la figura de los presidentes, que encuentran objetivos comunes y que consideran que la cooperación son el mejor camino para conjuntar esfuerzos, los objetivos que emanan de estos organismos regionales, no distan mucho de lo que al interior de la nación se planea o se pretende alcanzar. Por tanto, en los bloques de integración, tanto MERCOSUR como Alianza del Pacífico, sus bases fundacionales, si bien son puramente económicas o comerciales, los contactos políticos, indudablemente son requeridos para legitimar su funcionamiento, esto quiere decir 
que, en ambos bloques, dependen de la base política y de la voluntad de los Estados que la conforman, para alcanzar objetivos más ambiciosos.

Al ser así, se reparten atribuciones, se jerarquizan autoridades, y se establecen sedes donde se institucionalizan los organismos, encaminados a tener una forma tal, que acoplen dichos elementos consensuados por la sociedad internacional, o por lo menos para evitar ir en contra de ellos. A pesar que estos organismos tienen su estructura física dentro de los países miembros que la conforman, su funcionamiento se encuentra en un espacio externo al propio Estado, pues sus objetivos circundan en un ámbito internacional, susceptible a cambios repentinos o procesos coyunturales, esos objetivos que se plasman en lo internacional se vinculan con lo interno cuando las instituciones internacionales colaboran con los Ministerios o dependencias gubernamentales.

De manera transversal, las dependencias gubernamentales fijan objetivos propios una vez planteados los macro-objetivos a alcanzar, y sus acciones se plasman en políticas públicas y programas que recaen en la sociedad; así como recursos asignados para ejecutar dichos programas, de los cuales se espera cumplan con la función de atender a las demandas sociales.

\section{Los límites de la interinstitucionalidad}

La interinstitucionalidad forma una red de cooperación entre instituciones, que aún es imprecisa, dónde empieza y hasta dónde puede actuar, debido a que no existe dentro del país una legislación, manual o reglamento que así lo declare. Entonces, si bien no existe algo que defina su interacción, si existe algo que la limite, pues los reglamentos internos de las dependencias gubernamentales prohíben explícitamente que por cuenta propia se generen vínculos con el exterior, sin antes consultar con el Ejecutivo o con la Cancillería, esto con la función de mantener un control en dichas interacciones y la jerarquización subordinada. Kliksberg (1994) habla precisamente sobre la modernización del Estado que permita que las instituciones puedan tener mayores atribuciones, aunque en la actualidad no es así, la coyuntura internacional plantea un esquema cercano a esta idea. En el presente la retroalimentación de objetivos locales no está pensada para llegar a estructuras de integración regional, pues se consideran locales, y por tanto responsabilidades soberanas del Estado.

Cuando las problemáticas locales, llegan a tener correlación en otras partes del mundo, es ahí donde su categoría se eleva a otro estatus. Con todo y considerando la globalización y la interdependencia entre los países, esto es cada vez más común entre las relaciones vecinales, regionales e internacionales. Por lo tanto, la realidad está superando la actuación de estos organismos, que reaccionan a eventualidades surgidas entre los países, sin ser planeadas para aminorar problemas o conflictos ulteriores.

Las instituciones gubernamentales tienen el contacto directo con la sociedad, es decir con el individuo, cuyo papel e importancia es exponencialmente tomado en cuenta como un factor de orden internacional (bajo parámetros claros y específicos), por tanto, sus demandas son atendidas por las instituciones de manera natural, y corren el riesgo de perderse en muchas ocasiones en esquemas burocráticos. Cuando las demandas internas llegan a ser fuertes, legítimas y hacen eco en la colectividad, los organismos de integración no tienen la estructura necesaria para atender de manera conjunta tópicos que son de orden nacional. El hecho está que la soberanía de cada Estado permite que problemas internos se resuelvan por cuenta propia. Sólo cuando llega a afectar la estabilidad de la región o lleguen a afectar interés de otras 
naciones, se activan los mecanismos de cooperación, con la intención de crear objetivos y por tanto acciones, para conjuntar esfuerzos que radiquen con el problema o por lo menos lo contengan.

La función de los organismos de integración se vuelve difusa cuando los problemas internos se interponen en el accionar de los organismos internacionales. Hablando de los ejemplos mencionados con antelación. En el MERCOSUR, siendo uno de los organismos más estructurados institucionalmente en América del Sur, éste no tiene cabida cuando alguno de los países miembros tiene dificultades internas, sobre todo cuando se trata de cuestiones fuera del orden económico, e incluso aunque así lo fuera.

Por tanto, las instituciones a pesar de tener una estructura interna bien definida, está limitada de acción. Al mismo tiempo la relación entre las instituciones internacionales, también están acotadas a tópicos preestablecidos por los miembros o pactados en acuerdos explícitos. De cualquier forma, aún son inexistentes los mecanismos que permitan una cooperación transversal, en el sentido que las experiencias nacionales faciliten adaptar modelos, sin que ellos interfieran con la soberanía del país, haciendo que exista una mejor cooperación entre Estados. Dejando la idea que ha prevalecido celosamente entre las naciones latinoamericanas, de mantener pausada su interacción con el exterior, en favor de resolver problemas del orden interno.

Lo que se puede afirmar respecto a la coordinación entre instituciones está dado por las interacciones entre los países, las cuales son tan comunes y tan fuertes que resulta contradictorio la idea de mantenerse al margen de lo internacional. Simplemente las estructuras institucionales aún no están pensadas para una cooperación que no se perciba como injerencista, y se vea más como una cooperación real de infraestructuras funcionales para resolver problemas.

\section{El peligro de la burocratización en las instituciones internacionales}

La burocracia es un elemento que debe ser considerado en el funcionamiento y entendimiento de las instituciones. No solamente se debe de apreciar como la colectividad de funcionarios o un sistema organizacional estructurado y definido; se puede entender a la burocracia como una institución central del sistema democrático.

La burocracia en el complejo sistema interno del Estado es requerida para la acción gubernamental, su buena función limita las "políticas oportunistas y fortalece la confianza de los actores en cuanto al cumplimiento pleno de los compromisos contraídos como parte de los acuerdos que se celebraron" (Zuvanic, 2010: 10). La inexistencia de un aparato burocrático estropea la acción gubernamental al no tener directrices claras de funcionamiento, lo que gradualmente conllevaría a una inestabilidad política con conflictos de acción y reacción de las propias instituciones.

En términos institucionales, la política exterior es dirigida desde el ejecutivo a través de sus facultades otorgadas a los ministerios de relaciones exteriores de cada país, esto quiere decir que la propia política exterior desarrolla las mismas dinámicas que cualquier otra institución dentro del Estado y por ende se agrupa en un marco burocrático. Algunos autores advierten que esta burocracia que es muy particular del Estado del cual emana, se transfiriendo mediante este medio de política exterior hacia otras organizaciones de alcance regional o internacional. Esto quiere decir que los organismos de alcance regional retoman las pautas que las instituciones internas tienen y las llevan a un margen de acción 
colectivo. El resultado de la burocracia, la política exterior y las instituciones de índole internacional no siempre tienen los resultados esperados y mucho menos "una perspectiva que se centra exclusivamente en las interacciones que tienen lugar en la burocracia puede llevar a ignorar tendencias que tienen lugar en el sistema internacional y que restringen o expanden las opciones disponibles" (Van Klaveren, 1992: 207).

En el proceso de toma de decisiones, una burocracia profesional y bien encaminada puede ser un canal en el que el tiempo político de un dirigente no colisione con coyunturas internas o políticas incipientes, pensando en una pauta presidencialista latinoamericana cuya amenaza siempre latente de centralismo impacte de manera directa cualquier decisión y restitución de la misma. La burocracia puede influir para dar un reforzamiento institucional, lo que traería a estructuras más sólidas y constantes en el tiempo. "En consecuencia, la burocracia debe verse como un actor clave para alentar acuerdos prolongados, especialmente por su capacidad para llevarlos a la práctica. La misma está subordinada al gobierno dentro del sistema constitucional" (Zuvanic, 2010: 10).

Bajo los supuestos que han sido mencionados en este texto, en su forma institucional, la burocracia se distingue como "un conjunto articulado de reglas y pautas de funcionamiento cuya finalidad es asegurar la continuidad, coherencia y relevancia de las políticas públicas, por un lado, y prevenir la discrecionalidad del ejercicio del poder público por otro" (Zuvanic, 2010: 10). El proceso de desarrollo en las instituciones de integración regional en el continente, es compatible con esquemas burocráticos bajo ciertos considerandos, la institucionalidad puede compaginar sus estructuras con procesos que alienten su funcionamiento y permitan un desarrollo integral en el perfeccionamiento comunicacional de las redes interinstitucionales, la pauta a seguir en la vinculación de una interinstitucionalidad burocrática tiene que estar bien definida, orientada, coordinada, estructurada y planificada.

La idea de los viejos vicios institucionales puede ser uno de los mayores retos a vencer. La burocracia que está inmersa en las estructuras estatales, puede ser un factor que atente contra el buen funcionamiento de las instituciones internacionales, pues su base actual, está en los compromisos y plazos definidos que forman parte de un orden mundial. Sin embargo, Crozier afirma que: "Toda la literatura posweberiana sobre la burocracia padece en efecto de una ambigüedad fundamental. Por un lado, la mayoría de los autores creen que el desarrollo de las organizaciones burocráticas responde al advenimiento de la racionalidad en el mundo moderno, y que la burocracia es por ese hecho intrínsecamente superior a todas las demás formas posibles de organización. Por otro lado, muchos autores, a menudo los mismos, consideran a las organizaciones como especies de leviatanes por cuyo medio se prepara la esclavización del género humano" (Crozier, 1974: 55).

Al mismo tiempo las instituciones internacionales adoptan formas muy parecidas a las nacionales. Evidentemente al no seguir a una sola autoridad suprema, sino a un cúmulo de personas que se reconocen entre iguales, el consenso es vital para su formación, pero no evita que las estructuras subordinadas a este Consenso, Consejo o Élite Política, este fuera de un orden burocrático. Y aunque la base de las instituciones está pensada para tener una jerarquía y división de funciones, la burocracia excesiva sería un lastre para el funcionamiento óptimo y la ejecución de objetivos, para los cuales ese organismo fue creado.

Pensando en los ejemplos del MERCOSUR y la Alianza del Pacífico, siendo esquemas institucionales muy diferentes, aún su nivel de burocracia no es equiparable a los existentes en los países miembros y sus estructuras gubernamentales. Para el caso del MERCOSUR en particular, este organismo corre este riesgo en su vasta institucionalidad, de ser impregnado por una burocracia excesiva; de hecho, es un asunto aún 
no resuelto por los dirigentes ni los estudiosos, que siguen de cerca la estructura funcional del organismo. Para el caso de la Alianza su estructura es todavía imprecisa y poco ajustada a los estándares y esquemas tradicionales de la institucionalidad, al no contar con una Secretaría, o un organismo de coordinación permanente, se mantiene una burocracia suave, pero funcional en sus niveles bajos.

Un excesivo esquema de burocratización en su adaptación en instituciones regionales puede desbalancear los parámetros de poder dentro de un organismo, haciendo que pueda privilegiar a unos países más que otros. Estos mecanismos, creados para una democracia entre las partes, pueden impregnarse de esquemas de burocratización viciados para detener procesos o ralentizar avances que entren en conflicto con intereses entre las partes. El dejar una burocracia sin parámetros establecidos y con atribuciones excesivas hace que puedan duplicarse esfuerzos y desaprovechar oportunidades, por lo que el cuidado de que dicha burocratización esté planeada discrecionalmente para mejorar la capacidad de acción, y no empeorarla, es fundamental para el desarrollo y avance de los propios organismos, pues ellos tendrán que enfrentar indiscutiblemente, a la burocracia interna de cada país.

\section{Conclusiones}

El papel que juegan las instituciones, en cualquiera de sus versiones, funge como un depositario de responsabilidades muy específicas y acotadas a un régimen jerárquico. El funcionamiento y razón de ser de estas instituciones tiene que ver con sus objetivos y la estructura que forma para cumplirlos.

En el desarrollo de estos procesos, se crean redes y nexos con otros tipos de actores y organismos que requieren mantener una relación directa o indirecta. Cuando esto ocurre se desarrollan otras formas de resolver ciertas situaciones, pero al mismo tiempo, estas instituciones deben de superar grandes retos que se presentan al establecer dichos nexos. Sobre todo, el respeto de las facultades y jerarquías para las cuales están planeadas, dentro del marco regulatorio del derecho interno y del derecho internacional, ninguna institución puede sobrepasar de sus atribuciones, al contrario, debe de aprovechar la fluidez que existe en la armonía entre el derecho interno y comunitario al momento de establecer acuerdos y compromisos.

Recordemos que las fuentes del derecho internacional como la costumbre, los tratados y los principios generales del derecho, son las formas directas que regularmente encontramos para formalizar dichas instituciones a nivel internacional bajo la pauta voluntaria de los Estados (pacta sunt servanda) (Barberis, 1982: 10). Asimismo, existen otras fuentes indirectas, como los actos de las organizaciones internacionales y los actos unilaterales de los Estados, aparte de los medios auxiliares, que son la doctrina, la jurisprudencia y la equidad.

Dentro de este último esquema podemos encontrar acuerdos interinstitucionales, que tiene una jerarquía menor, estos son "memorándums de entendimiento" entre instituciones que sólo son vinculantes entre sí, y no afectan otras estructuras dentro del aparato gubernamental, es decir que su campo de acción tiene esa libertad de poder celebrar entre instituciones de distintos países, algunos acuerdos que se estipulan bajo un marco muy delimitado dentro del derecho. Pese a que estos memorándums de entendimiento o acuerdos interinstitucionales no tienen una vinculación con todo el Estado y su jurisdicción sólo compromete a ciertas áreas de las instituciones firmantes, son un precedente importante en el ejercicio de la paradiplomacia. En términos reales son la antesala para la ejecución de tratados más sólidos y comprometidos, pues su existencia permite a las instituciones establecer un mecanismo de acción y 
funcionamiento que mejora con la adopción de políticas públicas, así como de pautas planificadas de política exterior de los Estados.

La institucionalidad hablando de un mundo cada vez más global e interdependiente, requiere de adecuar esquemas que circundan lo interno y en lo internacional, estructuras que tienen lazos fuertes, pero que tratan de mantener una línea divisoria en sus jurisdicciones, atribuciones y jerarquías. La institucionalidad funciona como ese vínculo que entrelaza la cooperación y la coordinación de información, así como las acciones entre instituciones. Las instituciones, sean de orden interno o internacional, tienen la responsabilidad de reaccionar con cierta expectativa, ante ciertas coyunturas. Como se ha visto, existen temas que pasan de manera transversal por instituciones, o por lo menos existe un flujo real de cooperación y de acciones que se plantea de forma natural.

Al establecer decisiones conjuntas y negociar acciones específicas, se genera un diálogo político, este hecho forja desde otra perspectiva la relación entre los países, pues tiende estrechar las relaciones entre ellos y a mejorar el vínculo existente. Por tanto, cuando se permite que las instituciones puedan interactuar más fluidamente y basar acciones que incluyan su participación conjunta, la relación de cooperación que se institucionaliza, se crea una cultura institucional, la cual evoluciona en una mejora notable y crea mecanismos de acción más elaborados, incluyentes y funcionales en el tiempo.

En efecto que la institucionalidad y su rol como vínculo entre países, puede mejorar la relación que exista entre ellos. La razón se fundamenta en la cooperación, las instituciones son atemporales y su estructura es fácilmente identificable y acotada a ciertas áreas de funcionamiento, la anhelada integración entre países puede estar presente en las formas de integración entre instituciones de carácter interno e internacional. Esto no quiere decir que se base en ella, sino que, bajo el mismo esquema acotado a objetivos y acciones, se le permita incursionar a otras áreas y a una mayor cantidad de participantes.

En la actualidad, se están viendo cada vez más proyectos que requieren de una participación de instituciones mayor a la existente, pero esto aún no es real para el contexto latinoamericano, pues el recelo de información y la necesidad de mantener alejados a los países vecinos por problemas internos, hasta que estos lleguen a ser parte de la problemática internacional, es parte de la expresión de la cultura del continente. El rol que juega la institucionalidad es una oportunidad para ampliar la cooperación entre los países que así lo decidan, pues la voluntad de relacionarse entre sí, y establecer metas reales, involucra elementos internos e internacionales de coordinación, que todavía se mantienen acotados a dependencias que tampoco permiten una interinstitucionalidad interna.

Entre instituciones internas e internacionales, puede haber elementos rescatables para establecer compromisos reales y acciones verdaderas, que puedan tener metas específicas y apoyo multilateral, así como recursos económicos que apoyen a cumplirlas. La capacidad de interferencia de organismos internacionales puede perfectamente funcionar limitando su participación a recomendaciones, apoyando a mejorar situaciones que recaen en un orden multilateral, sin que ello interfiera en la soberanía estatal ni propicie una injerencia indiscriminada.

El resultado del análisis apunta al hecho que la integración regional en América Latina requiere innovar en sus acciones, los países buscan constantemente formas de mantener y mejorar los vínculos permanentes entre los Estados. En la mayoría de los casos, la cooperación está basada en la diplomacia, y esta, aunque es muy importante, no es la única que puede mejorar los contactos a otros niveles. En las últimas décadas hemos sido testigos de otras formas de contacto entre países, algunos de ellos como la 
paradiplomacia, los negocios internacionales y los encuentros interparlamentarios. Todos ellos desde sus áreas crean estructuras y diálogos que se plasman en avances significativos y un aporte extraordinario a la relación entre países. La integración y avance en la relación regional, puede estar apoyada en las instituciones, pues ellas mantienen contactos y vínculos de cooperación, entre tipos de instituciones que son de orden interno e internacional.

El verdadero aporte está en la relación que exista entre ellos y su forma de accionar ante coyunturas específicas, ya que los Estados pueden enfrentar retos de mejor forma de manera conjunta que individualmente. Sin embargo, los mismos Estados, aún no tiene presente los alcances de hacer más participativa a las instituciones en la cooperación internacional y el desarrollo de la integración entre países, las legislaciones, tratados, y normatividad no está pensada para este hecho en lo inmediato.

Puede que esta precaución o falta de incursión ante una coordinación institucional, demerite algunos esfuerzos importantes y oportunidades que se dejan pasar. Indudablemente que en el mediano plazo esta relación interinstitucional, superará los descuidos del Estado moderno para incluir la participación de instituciones en la integración de países, pues sus contactos están guiándose cada vez más frecuentemente por la línea de temas, que por la inclusión de los países. Algunos objetivos son demandados por las naciones en un orden mundial, y estos requieren de reaccionar, para no quedarse aislados o demostrar a nivel de imagen internacional, que están trabajando en pro de temas que involucren a todos. Siendo así, las estructuras internas se ven comprometidas a entablar y mantener contactos con otras instituciones, sus objetivos van en función de optimizar sus capacidades de acción.

Por ejemplo, el tema del terrorismo obliga a las naciones a reaccionar ante este fenómeno, por lo que las estructuras internas requieren de mantener contactos internacionales con sus pares en otras naciones, para hacer fluir información que permita la prevención de ataques. Ante la falta de coordinación y participación entre instituciones, es imposible el combate a esta problemática, atentando contra la población civil y mostrando la incapacidad del Estado de velar por el bien internacional, así como mantener la seguridad interna y salvaguarda de la población.

Todo lo anterior permite definir la importancia de una interinstitucionalidad armoniosa, con las características delimitadas a sus objetivos, con las atribuciones necesarias para su funcionamiento y eventual desenvolvimiento, la cooperación, como hemos evidenciado, requiere de formas más innovadoras de acción conjunta, de la inclusión de estructuras atemporales y consolidadas, que den certeza y confianza. La interinstitucionalidad profundiza la forma en que los Estados pueden aprovechar sus elementos burocráticos y estructuras afines. La fluidez en función de la voluntad política tiene menor posibilidad de estancamiento ante esquemas de trabajo consolidados. La interinstitucionalidad en cualquiera de sus formas, alienta un desarrollo próspero de cooperación en distintos niveles y diversas estructuras, su evaluación es positiva y en ningún caso va en contra de un avance del desarrollo integracionista latinoamericano. 
Latin American Journal of Trade Policy 3 (2019) - Universidad de Chile

\section{Referencias}

Allard, R. (2009), La globalización por dentro: el Estado-Nación y los actores transnacionales. Santiago de Chile: Catalonia.

Allard, R. y Wilhelmy, M. (2017), América Latina y Chile en el mundo global. Algunas tendencias en el siglo XXI. Burgos, Mauricio. Desafíos de la diplomacia parlamentaria y estudio de caso sobre la Alianza del Pacífico. Pontificia Universidad Católica de Valparaíso. Valparaíso. Chile.

Barberis, JA. (1982), El Concepto De Tratado Internacional. Anuario de derecho internacional. VI.

Bernales, E. (1999), Crítica al presidencialismo en América Latina. Revista Iberoamericana de Filosofía, Política, Humanidades y Relaciones Internacionales. Araucaria. Vol. 1, núm. 2.

Castedo, L. (2008), Fundamentos culturales de la integración latinoamericana, Dolmen ensayo, Volumen 33 de Encuentros (Banco Interamericano de Desarrollo) Washington, D.C.

Campo, F. (2017), CEPAL, Naciones Unidas. Acciones estratégicas para profundizar la integración económica en Centroamérica. Sede subregional en México. Publicaciones de Naciones Unidas. México.

Colacrai, M. (2006), La marcha de la integración en América Latina. El rol de las ideas, instituciones y políticas en el MERCOSUR. En, Política y movimientos sociales en un mundo hegemónico. Lecciones desde África, Asia y América Latina. CLACSO, Consejo Latinoamericano de Ciencias Sociales. Buenos Aires. Argentina. Disponible en: http://bibliotecavirtual.clacso.org.ar/clacso/sur-sur/20100711043848/16_PIVCtres.pdf

Crane, K. Gramlich, M. y Peterson, K. (2004), Putting Interagency Agreements into Action. Issue Brief, Examining Current Challenges in Secondary Education and Transition. Issue 2. Vol. 3. September 2004. Disponible en : http://ncset.org/publications/issue/NCSETIssueBrief_3.2.pdf

Crisorio, C. (1974), Historia y perspectiva de la integración latinoamericana, The University of Michigan, Asociación por la Unidad de Nuestra América (Cuba), 2000.

Cunill, N. (2013), La intersectorialidad en las nuevas políticas sociales: Un acercamiento analíticoconceptual.

Crozier, M. (1974), El fenómeno burocrático: ensayo sobre las tendencias burocráticas de los sistemas de organización modernos y sus relaciones con el sistema social y cultural. Amorrortu editores, Buenos Aires, Argentina.

---- (1984), No se cambia la sociedad por decreto. Instituto Nacional de Administración Pública. ISSN: 1989-8983 - Depósito legal: M-581-1958

Duque, J. (2011), El desafío de la interinstitucionalidad como estrategia de gestión en programas de formación avanzada. Revista Vol.11 No.3. Versión Digital. Facultad de Educación. Universidad de Antioquia. Medellín, Colombia.

Duverger, Maurice (1970) Instituciones políticas y derecho constitucional. Barcelona, Ariel.

García, R. (1999), Derecho comunitario y Derechos nacionales: autonomía, integración e interacción. Prólogo de Juan Carlos Cassange. Argentina. Abeleto-Perrot.

Guillen, A. (2007), Economía y Sociedad en América Latina: entre la globalización, la regionalización y el cambio estructural. México: UAM, unidad Iztapalapa, Porrúa. 325.

Hofstede, G. (2010), Cultures and Organizations: intercultural cooperation and its importance for survival. 3 edition, New York, McGraw-Hill.

Jentleson, B. (2014). American Foreign Policy. The Dinamycs of Choise in the21st Century.

Kliksberg, B. (1994), El rediseño del Estado una perspectiva internacional. Instituto Nacional de Administración Pública. Fondo de Cultura Económica. México. 
Mora, J. (2016), La Alianza del Pacífico y Mercosur: evidencias de convergencia económica. Estudios Gerenciales 32 (2016) 309-318. Calí, Colombia. Universidad ICESI.

North, D. (1993), Instituciones, cambio institucional y desempeño económico. Fondo De Cultura Económica.

Pizarro, C. (2016), Immanuel Wallerstein: Globalización de la economía-mundo capitalista. Perspectiva a largo plazo. Fondo De Cultura Económica. Chile.

Repetto, F. (2004), Capacidad Estatal: requisito para el mejoramiento de la Política Social en América Latina. Departamento de Integración y Programas Regionales Instituto Interamericano para el Desarrollo Social. Washington D.C.

Rocca, M. (2010), Manual de la Teoría del Estado. Editorial Forja. Santiago de Chile.

Sanahuja, J. et al. Sección I La reconfiguración de América Latina y el Caribe: ¿Regionalismo post-liberal, post-hegemónico o post- neoliberal? El debate en curso. Anuario de Integración 9. Buenos Aires, Argentina.

Serbin. A. el al. (2012), El regionalismo "post-liberal” en América Latina y el Caribe: Nuevos actores, nuevos temas, nuevos desafíos Anuario de la Integración Regional de América Latina y el Gran Caribe 2012.

Tamames, R. (2012), La Economía Internacional en el Siglo XXI, colección de estudios socioeconómicos, Mediterráneo Económico 22, Fundación Cajamar, España.

Van Klaveren, A. (2012). América Latina en un nuevo mundo. Revista CIDOB d'Afers Internacionals 100, 2012, pp. 131-150.

---- (1992). Entendiendo las políticas exteriores latinoamericanas: modelo para armar. Estudios Internacionales, VOL. 25 NÚM. 98. p. 169-216.

Winer \& Ray, (1994), Hacia un modelo de coordinación interinstitucional e intersectorial para disminuir el trabajo infantil agrícola en México. La coordinación interinstitucional e intersectorial: un diagnóstico situacional para mejorar la eficiencia de la colaboración coordinada entre el sector social y las instituciones del Estado Mexicano.

Waltz, K. (1988), Teoría de la política internacional. Grupo Editor Latinoamericano.

Weber, M. (1977), ¿Qué es la Burocracia? Alemania. Recuperado de la web, 18 de enero 2018: Disponible en http://www.ucema.edu.ar/u/ame/Weber_burocracia.pdf

Zuvanic, L y Iacoviello, M. (2010), La burocracia en América Latina. ICAP-Revista Centroamericana de Administración Pública (58-59): 9-41.

\section{Recursos electrónicos}

Banco Interamericano de Desarrollo (BID); Organización para la Cooperación y el Desarrollo Económico. Panorama de las Administraciones Públicas: América Latina y el Caribe 2017.

Banco Mundial. (2017), El futuro de la integración latinoamericana, página oficial en la web: http://www.bancomundial.org/es/events/2017/03/08/el-futuro-de-la-integracionlatinoamericana

CEPAL, Comisión Económica para América Latina y el Caribe (2014), La Alianza del Pacífico y el MERCOSUR: hacia la convergencia en la diversidad.

----(2014), Naciones Unidas. Panorama de la inserción internacional de América Latina y el Caribe. Integración regional y cadenas de valor en un escenario externo desafiante. Capítulo III, El 
Aporte de la integración regional a la integración productiva. Publicación de las Naciones unidas. Santiago de Chile. Pág. 89-101.

---- (2018), Institutional frameworks for social policy in Latin America and the Caribbean. Editor Rodrigo Martínez. ECLAC Books. Printed at United Nations, Santiago. S.18-00484.

---- (2018). Perspectivas del Comercio Internacional de América Latina y el Caribe: Las tensiones comerciales exigen una mayor integración regional. ISBN: 978-92-1-058637-5 Impreso en Naciones Unidas, Santiago. S.18-01070.

Centro Coordinador y Difusor de Estudios Latinoamericanos. (2006), América Latina las caras de la diversidad, México, D.F. UNAM, Centro Coordinador y Difusor de Estudios Latinoamericanos.

DIRECON, Dirección General de Relaciones Económicas Internacionales de Chile https://www.direcon.gob.cl

----(2016), Acuerdos Comerciales, Gobierno de Chile y Objetivos, 14-11-16, Recuperado de: https://www.direcon.gob.cl/acuerdos-comerciales/

INDESOL, Instituto Nacional de Desarrollo Social. (2011), Hacia un modelo de coordinación interinstitucional e intersectorial para disminuir el trabajo infantil agrícola en México. Reflexiones y praxis desde los actores sociales e institucionales En el marco de la Convocatoria 2011: https://www.unicef.org/mexico/spanish/mx_02I-Hacia_un_modelo_de_coordinacion...pdf

La Razón. Diario periodístico de publicación nacional. Lima, Perú En la web: http://larazon.com/mundo/Fiscales-America-Latina-Lima-trasnacionales_0_2463353730.html

Página oficial de Alianza del Pacífico. https://alianzA.P.acifico.net/

Página oficial de CELAC. http://www.sela.org/celac/quienes-somos/que-es-la-celac/

Página oficial de OEA. http://www.oas.org/es/

Panorama de las Administraciones Públicas América Latina y el Caribe. 2017: https://publications.iadb.org/bitstream/handle/11319/7979/Panorama-de-las-

Administraciones-Publicas-America-Latina-y-el-Caribe-2017.pdf

Programa Iberoamericano para el Fortalecimiento de la Cooperación Sur- Sur. Archivo de Noticias Chile. Chile contará con una política y estrategia de Cooperación para el Desarrollo. 2013 